\title{
¿Qué investigan sobre emprendurismo las Instituciones de Educación Superior Pública en Oaxaca?
}

\author{
Javier Damián Simón \\ Bertha López Azamar \\ Flor Garza Vargas \\ Guadalupe Estela Peralta Santiago \\ Universidad del Papaloapan, campus Tuxtepec
}

\section{Resumen}

ste trabajo forma parte de una $\checkmark$ investigación titulada "Estado del arte de la investigación sobre educación, derecho, género y emprendurismo, efectuada en las instituciones de educación superior pública y otras instituciones de la sociedad del estado de Oaxaca, periodo 2005-2011. Análisis, perspectivas y propuestas", financiada por el Promer en el marco del programa de Consolidación de Cuerpos Académicos.

En este artículo se presentan algunos resultados obtenidos de la elaboración del estado del arte de la investigación sobre la temática de emprendurismo producida por los profesores adscritos en las Universidades e Instituciones de Educación Superior Pública del Estado de Oaxaca. Los resultados muestran que la producción sobre la temática se encuentra en desarrollo y que es necesario hacer esfuerzos para consolidarla por tres razones: solo en la tercera parte de las 34 instituciones estudiadas se efectúa investigación sobre emprendurismo, a pesar de que en la mayoría se ofertan carreras sobre negocios y dirección de empresas, además, de los trece campos temáticos que los especialistas proponen para clasificar este tipo de investigación solo se ha indagado en nueve de ellos existiendo un vacío en cuatro campos lo que representa grandes oportunidades para investigaciones futuras. Se encontró que se ha privilegiado la investigación en emprendurismo lucrativo, escasamente se ha atendido el emprendurismo social y femenino y en menor medida se han efectuado trabajos de investigación sobre emprendurismo innovador.

Palabras clave: estado del arte, investigación, educación superior, emprendurismo. 


\section{Introducción}

La investigación sobre emprendurismo es un tema que actualmente está cobrando importancia entre la comunidad académica de las universidades e instituciones de educación superior, en particular en aquellas que ofertan programas educativos de corte económico administrativo y/o de tipo empresarial (González, Peña y Vega, 2010); lo anterior originado en parte por el papel protagónico que se le ha asignado al emprendurismo en la creación y puesta en marcha de unidades económicas así como en la generación de empleos.

El tema del emprendurismo es todavía un campo de estudio e investigación en proceso de exploración, según Watson (2001) este tema es un campo de estudio joven y consecuentemente presenta para los académicos investigadores problemas y retos tales como la definición de marcos conceptuales y de términos homologados entre todos los interesados; para su estudio resulta necesario estandarizar la investigación en emprendurismo con parámetros específicos tal como se hace en otros campos de la ciencia, definiciones precisas, recolección de datos de forma objetiva, replicabilidad de los hallazgos, abordaje sistemático y acumulativo; cuyos propósitos -ya sean para la explicación, predicción y comprensión de una realidad- se deriven en una teoría, la cual está todavía en proceso de construcción en el campo del emprendimiento.

Por lo tanto, para analizar e investigar al emprendurismo es necesario entenderlo e interpretarlo desde diversas perspectivas que establezcan nuevas formas para estu- diarlo en su complejidad, construyendo un discurso inter y transdisciplinario. Lo anterior es un proceso indispensable, debido a que en últimas fechas hay personas que efectúan investigación sobre temas de emprendurismo a pesar de no contar con una formación en el área de empresas y negocios como generalmente se esperaría; en relación con lo anterior Villa et al (2007) dicen que el tema del emprendurismo ha sido tomado como objeto de investigación en otras áreas del conocimiento como la sociología, la historia, la sicología, la comunicación y otras áreas afines a la economía.

En otros estudios se encontró una alta permeabilidad en el tema del emprendimiento con autores de otras disciplinas, según Harrison y Leitch (1996) se introduce la especialización acumulada al posibilitar la aplicación de conocimientos disciplinares a situaciones de emprendimiento, que a la vez son la semilla sobre la que se construye un nuevo campo de conocimiento del fenómeno emprendedor que requiere la elaboración teórica a partir de diversas aproximaciones multidisciplinarias (Bygrave, 1989). Se entiende entonces que investigar el tema del emprendimiento no puede concebirse solo desde el concepto de oportunidad, los rasgos del individuo y sus capacidades para lograr la efectividad, más bien, integrando todas las categorías mencionadas lo que brindará la oportunidad de identificar nuevas áreas praxeológicas, epistemológicas, axiológicas y ontológicas.

A pesar de que se reconoce la importancia del emprendurismo, son muy escasos los trabajos sobre el estado del arte o estado del conocimiento de las investigaciones 
generadas sobre el tema. Generalmente, las investigaciones se han enfocado en dos sentidos, primeramente en estudiar el potencial de los individuos para ser emprendedores y comparar dichos resultados entre países y regiones tal como lo muestra el Informe GEM España 2010, que lidera el IE Business School (Kelley, Bosma y Amorós, 2011) y la investigación patrocinada por el Banco de Desarrollo de América Latina llevada a cabo en Nicaragua, Honduras, Guatemala, Panamá, El Salvador, Costa Rica y República Dominicana (Reyes, Zilleruelo e Hidalgo, 2012).

En el mismo orden de ideas la Comisión Europea a través del Programa TouriSME, llevó a cabo una evaluación comparativa de la situación que guardaba el emprendurismo en cuatro regiones de la Unión Europea, Comunidad Valenciana, North East of England, Sachsen-Anhalt y, Limburg (García, Villanueva y Collado, 2005). En segundo lugar, existen otras investigaciones que tienen como principal objetivo recopilar las diversas acciones, políticas, estrategias, prácticas y procedimientos que se están desarrollando o impulsando desde el ámbito escolar a fin de lograr una compilación de buenas prácticas para promover las actitudes y capacidades empresariales mediante la educación (CE, 2004; Pérez, 2010; Valls, Villa, Martínez y Hernando, 2009) aunque hay que aclarar que ya se han efectuado en países de Europa.

En México, escasamente se conoce el desarrollo, el grado de avance y consolidación de la investigación sobre la temática efectuada en las Instituciones de Educación Superior (IES en adelante); a la fecha no existe en nuestro país un inventario, estado del arte y/o estado del conocimiento de la investigación sobre emprendurismo lo que ha ocasionado un desconocimiento de cuáles son los temas más recurrentes, los menos trabajados, así como las líneas de investigación emergentes sobre el tema. En este trabajo de investigación se realizó un análisis de la producción académica y los resultados obtenidos mostraron que en las IES públicas del estado de Oaxaca, donde existe una gran necesidad por fortalecer esta área del conocimiento no se han generado estudios que den cuenta sobre las problemáticas que se han atendido ni del grado de avance o consolidación del emprendurismo como objeto de estudio, a pesar de que la mayoría de las IES públicas del estado ofertan programas educativos a nivel licenciatura tales como Administración de Empresas, Gestión y Dirección de Negocios, Comercio Internacional, Mercadotecnia, Contaduría, entre las más representativas, así como algunos programas de posgrado en área de Administración de empresas y negocios.

El objetivo principal del presente trabajo fue elaborar el estado del arte de la investigación relacionada con estudios sobre el emprendurismo efectuada en las IES públicas del estado de Oaxaca durante el periodo de 2005 al 2011, para lo cual se establecieron cuatro objetivos particulares:

1) Elaborar un estado del conocimiento sobre la investigación en emprendurismo a fin de identificar las temáticas de investigación más recurrentes, las áreas emergentes y los vacíos de investigación que merecen ser atendidas. 
2) Identificar la fase en la que se encuentran dichos trabajos, las regiones del estado en donde se desarrollan y los problemas que se han afrontado para llevarlas a cabo.

3) Socializar los resultados con los investigadores de las IES públicas, con el fin de que sean de utilidad para sus trabajos de investigación, así como para los estudiantes que cursen algún programa de maestría y doctorado en la temática.

4) Conformar grupos o redes de investigadores con temáticas afines al emprendurismo, que permitan fortalecer e impulsar la investigación de primer nivel en las IES públicas del estado de Oaxaca.

\section{Metodología}

El tipo de estudio fue teórico, documental, descriptivo y exploratorio en el cual se utilizó un enfoque cuanti-cualitativo para poder describir la producción académica de los investigadores adscritos a las IES públicas del estado de Oaxaca. El universo de estudio fue toda la producción escrita por los profesores-investigadores adscritos en las 34 IES públicas del estado de Oaxaca, en forma de memorias de congresos, foros, coloquios, ensayos, tesis de posgrado, artículos en revistas, capítulos de libro y, libros que aborden la temática de emprendurismo durante el periodo 2005-2011. Para integrar el estado del arte sobre emprendurismo se consiguieron los productos académicos generados con esta temática en el periodo mencionado, siguiendo las siguientes etapas:

a) Se obtuvieron las memorias escritas del $11^{\circ}, 12^{\circ}$ y $13^{\circ}$ Foro Estatal de In- vestigación e Innovación organizados por el Consejo Oaxaqueño de Ciencia y Tecnología (Сосут) en 2006, 2010 y 2011.

b) Se consultaron las páginas web de todas las universidades pertenecientes al Sistema de Universidades Estatales del Estado de Oaxaca -11en total-, pues en éstas aparecen los nombres y correos electrónicos de los profesores investigadores adscritos así como un breve $\mathrm{CV}$ con sus últimas producciones.

c) La misma acción se llevó a cabo en otras IES públicas, de las cuales solo fue posible obtener información de la página del Cimdir-Ipn Oaxaca y del Instituto Tecnológico de Oaxaca.

d) Se revisaron las únicas tres revistas de divulgación científica editadas por IES del estado para ubicar artículos sobre emprendurismo: Temas de Ciencia y Tecnología editada por la Universidad Tecnológica de la Mixteca, Ciencia y Mar editada por la Universidad del Mar, y Naturaleza y Desarrollo editada por el Instituto Tecnológico de Oaxaca.

e) Se consultaron dos libros editados por el SunEo: "La investigación científica en el Estado de Oaxaca" y "La verdad de los hechos", mismos que contienen los nombres de los profesores investigadores adscritos a las IES de este sistema y los títulos de su producción académica generada en los últimos años.

f) Con el nombre del investigador y títulos de su producción, se utilizó el 
REVISTA DE INVESTIGACIÓN EDUCATIVA DE LA REDIECH N. 9

ISSN: 2007-4336

motor de búsqueda de Google académico y el buscador Biblat mediante los cuales se recuperó la mayoría de la producción académica.

Obtenidos los 60 trabajos, se procedió a su lectura, organización y sistematización según la IES de adscripción de los investigadores, tipo de producción científica, campos temáticos que proponen Villa $\mathrm{y}$ colaboradores y tipo de emprendurismo estudiado. Puesto que el análisis del em- prendimiento debe hacerse a través de los diversos aportes teóricos y que para su comprensión e interpretación es necesario estudiarlo de manera inter y transdisciplinaria, se aplicaron distintos enfoques disciplinares al tema del emprendimiento, por ello, para sistematizar la información de la producción recabada se tomaron los 13 campos temáticos que proponen Villa et al (2007); dichos campos temáticos se enlistan y describen brevemente en la tabla 1 :

Tabla 1. Los campos temáticos de la investigación sobre emprendurismo

Campo temático

1. Educación para la formación de emprendedores

2. Métodos para el impulso a la creación de empresas

3. Investigación de género y grupos

4. Estudios de oportunidad

5. Condiciones económicas, culturales y/o sociales

6. Características personales del emprendedor

7. Tipo de organizaciones que crean los emprendedores

8. Buenas prácticas empresariales

9. Línea histórica

10. Emprendimientos sociales

11. Fuentes de financiación para las nuevas empresas

12. Emprendimientos en empresas intensivas en tecnología

13. Estudios comparativos
Descripción

Investigaciones sobre procesos pedagógicos y de enseñanza para formar y potencializar las habilidades para el emprendurismo empresarial, lo que incluye la instrucción en aspectos económicos, sicológicos, sociales, históricos y técnicos para formar profesionales capaces de crear empresas.

Metodologías para promover el emprendurismo empresarial desde la docencia como del apoyo a los emprendedores.

Investigaciones referidas al emprendurismo de la mujer, grupos sociales como jubilados, discapacitados, raciales o étnicos, jóvenes y en general los que clasifiquen en la definición de género.

Las oportunidades de generación de empresas que se desarrollen en los estudiantes y comunidad, la habilidad para identificar y distinguir posibilidades de creación de empresas que aporten a la cadena productiva de bienes y servicios.

Trabajos que evalúen la incidencia de las condiciones socioeconómicas en las cuales se establecen los nuevos emprendimientos, efecto ejercido por el medio.

Investigaciones para determinar las características o perfiles de los empresarios y, la identificación de la presencia o no de éstas en los grupos de población atendidos.

Investigaciones según el tipo de organizaciones constituidas por los emprendedores (PyMES, MiPyMES, empresas familiares), con o sin ánimo de lucro, asociativo, entre otras.

Trabajos que aborden diferentes prácticas exitosas de gestión o producción en los nuevos emprendimientos; aquellas actuaciones que suponen una transformación en las formas y procesos de actuación que son un germen para el cambio positivo en los métodos de actuación tradicionales. La labor del emprendedor y el empresario en busca de generar un clima de reconocimiento, documentar los diferentes casos y transmitir la experiencia vivida por los emprendedores.

Emprendurismo donde se prioriza la construcción de trama social en comunidades, regiones o grupos, con miras a generar emprendimientos empresariales en los cuales se construya capital social con sostenibilidad económica.

Identificación de la existencia de fuentes de financiación y el impacto logrado por éstas.

Línea que caracteriza a las empresas con alto componente tecnológico, empresas pequeñas, flexibles, con gran capital intelectual y estructuras y relaciones administrativas basadas en el conocimiento.

Integra los análisis comparativos sobre el emprendurismo entre regiones, países o grupos poblacionales.

Fuente: Tomado y adaptado de Villa et al (2007)

OCTUBRE 2014 —MARZO 2015 
Aunado a los campos temáticos anteriores, otros estudiosos clasifican la producción en emprendurismo en cinco grupos, mismos que fueron utilizados en este trabajo entre los cuales se encuentran el emprendurismo tradicional (Kihilstrom y Laffont 1979; Casson 1982; Veciana 1999), el emprendurismo social (De Pablo, 2005), el emprendurismo femenino (Fuentes y Sánchez, 2010), el emprendurismo innovador (Casson, 1982) y el emprendurismo de innovación social (Harrison y Leitch, 1996).

\section{Resultados de la investigación}

Atendiendo a la riqueza de los resultados obtenidos y considerando los criterios de espacio de esta publicación, en este apartado se describen brevemente aquellos aspectos que consideramos más relevantes sin que lo anterior signifique que no entrar en detalles o en una discusión amplia minimice la comunicación de los hallazgos de la investigación.
1. Características de los investigadores según la Institución de Educación Superior de adscripción

Al efectuar el análisis y la clasificación de los diversos documentos según IES (tabla 2), se encontró que 96 profesores reportaron producción escrita sobre emprendurismo (63 hombres y 33 mujeres) estos profesores están adscritos en 12 de las 34 IES públicas del Estado (35\% del total) a pesar de que en la mayoría de las 34 instituciones públicas se imparten programas de licenciatura en áreas relacionadas con las empresas o los negocios y en algunas estudios de posgrado. Tomando en cuenta el número de profesores adscritos destacan cuatro IES públicas: la Universidad del Mar (15), el CiIdir-IPN (14), la Universidad del Papaloapan (12) y el Instituto Tecnológico de Oaxaca (10); se observa que las IES con escasos recursos humanos que investigan la temática son el Instituto Tecnológico de Comitancillo con dos investigadores y el Instituto Tecnológico Agropecuario de Oaxaca con un profesor adscrito. Estos resultados muestran que falta hacer esfuerzos para que los profesores de estas IES efectúen investigación sobre emprendurismo. 
REVISTA DE INVESTIGACIÓN EDUCATIVA DE LA REDIECH N. 9

ISSN: 2007-4336

\section{Tabla 2. IES y profesores que han investigado temas de emprendurismo}

\begin{tabular}{|c|c|c|c|c|}
\hline Institución de Educación Superior & $\begin{array}{l}\text { Programas educativos donde se ha investigado em- } \\
\text { prendurismo }\end{array}$ & $\begin{array}{c}\text { No. de in- } \\
\text { vestigadores }\end{array}$ & Total & \\
\hline & & Hombres & Mujeres & \\
\hline Universidad del Mar & $\begin{array}{l}\text { Lic. en Administración Turística } \\
\text { Maestría en Mercadotecnia Turística }\end{array}$ & 9 & 6 & 15 \\
\hline $\begin{array}{l}\text { Centro Interdisciplinario de In- } \\
\text { vestigación para el Desarrollo In- } \\
\text { tegral Regional-IPN }\end{array}$ & $\begin{array}{l}\text { Maestría y Doctorado en Ciencias, área Administra- } \\
\text { ción y Conservación de Recursos Naturales }\end{array}$ & 6 & 8 & 14 \\
\hline Universidad del Papaloapan & Lic. en Ciencias Empresariales & 8 & 4 & 12 \\
\hline Instituto Tecnológico de Oaxaca & $\begin{array}{l}\text { Ing. en Gestión Empresarial } \\
\text { Lic. en Administración } \\
\text { Maestría en Administración } \\
\text { Maestría y Doctorado en Ciencias en Desarrollo Re- } \\
\text { gional y Tecnológico }\end{array}$ & 8 & 2 & 10 \\
\hline $\begin{array}{l}\text { Universidad Autónoma Benito } \\
\text { Juárez de Oaxaca }\end{array}$ & $\begin{array}{l}\text { Lic. en Administración } \\
\text { Lic. en Ciencias Empresariales } \\
\text { Lic. en Turismo y Desarrollo Sustentable } \\
\text { Lic. en Economía } \\
\text { Maestría y Doctorado en Ciencias Administrativas }\end{array}$ & 5 & 4 & 9 \\
\hline $\begin{array}{l}\text { Instituto Tecnológico del Valle de } \\
\text { Oaxaca }\end{array}$ & $\begin{array}{l}\text { Ing. en Agronomía (Agroindustrias y Producción } \\
\text { Pecuaria) } \\
\text { Ing. Forestal (Manejo de Recursos Forestales) } \\
\text { Ing. en Desarrollo Comunitario }\end{array}$ & 8 & 1 & 9 \\
\hline Universidad del Istmo & Lic. en Ciencias Empresariales & 8 & - & 8 \\
\hline Universidad de la Sierra Juárez & $\begin{array}{l}\text { Ing. Forestal } \\
\text { Maestría en Ciencias en Conservación de los Recur- } \\
\text { sos Forestales }\end{array}$ & 4 & 3 & 7 \\
\hline $\begin{array}{l}\text { Universidad Tecnológica de la } \\
\text { Mixteca }\end{array}$ & $\begin{array}{l}\text { Lic. en Ciencias Empresariales } \\
\text { Maestría en Administración de Negocios }\end{array}$ & 2 & 2 & 4 \\
\hline Universidad de la Sierra Sur & Lic. en Ciencias Empresariales & - & 3 & 3 \\
\hline $\begin{array}{l}\text { Instituto Tecnológico de Comi- } \\
\text { tancillo }\end{array}$ & $\begin{array}{l}\text { Ing. en Gestión Empresarial } \\
\text { Ing. en Agronomía }\end{array}$ & 2 & - & 2 \\
\hline $\begin{array}{l}\text { Centro de Bachillerato Tecnológi- } \\
\text { co Agropecuario* }\end{array}$ & Técnico Agropecuario & 2 & - & 2 \\
\hline $\begin{array}{l}\text { Instituto Tecnológico Agropecua- } \\
\text { rio de Oaxaca }\end{array}$ & $\begin{array}{l}\text { Ing. en Agronomía } \\
\text { Ing. Forestal } \\
\text { Maestría en Ciencias en Productividad de Agroeco- } \\
\text { sistemas }\end{array}$ & 1 & - & 1 \\
\hline TOTALES & & 63 & 33 & 96 \\
\hline
\end{tabular}

*Se encontró un trabajo de un profesor de nivel bachillerato cuya habilitación es de doctor en ciencias 


\section{Tipo de publicación producida}

(artículos, libros, ensayos).

Los resultados de la gráfica 1 muestran que 28 trabajos (47\%) fueron ponencias publicadas en forma de memorias en extenso y presentados en su mayoría en foros y eventos académicos estatales (Oaxaca) regionales (Tabasco y Puebla) e internacionales (Distrito Federal); 26 (43\%) fueron publicados en forma de artículos en revistas tanto nacionales como extranjeras en idioma español; 3 (6\%) fueron tesis de licenciatura, un ensayo (2\%) y un capítulo de libro (2\%). Durante el periodo analizado no se encontraron libros editados y producidos sobre la temática en ninguna de las IES oaxaqueñas. La cantidad de ar- tículos publicados pueden indicar que si bien el tema de emprendurismo es escasamente abordado por las 34 IES públicas la producción existente reúne los requisitos de calidad al aprobar la evaluación de pares en el proceso de dictamen de las revistas en donde han sido publicados los trabajos, de igual forma el número de trabajos presentados en los foros y congresos muestra el potencial que se tiene para que en el futuro los profesores investigadores "afinen" los resultados obtenidos y puedan publicarlos en forma de artículos de revistas, ensayos, capítulos de libros o libros pues las tres últimas modalidades de producción es muy escasa y en algunos casos fue inexistente en los materiales recopilados para esta investigación.

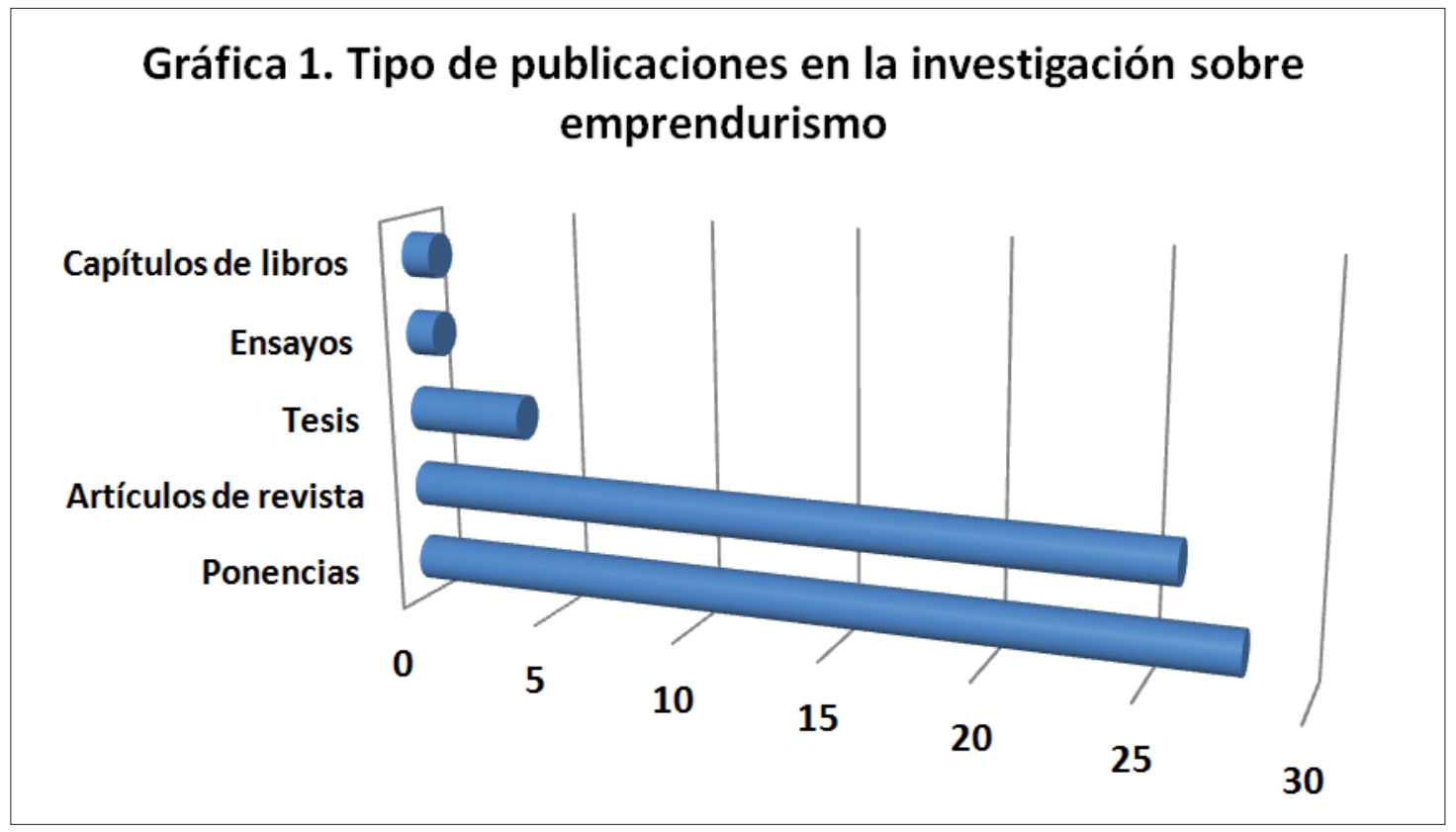


REVISTA DE INVESTIGACIÓN EDUCATIVA DE LA REDIECH N. 9

ISSN: 2007-4336

3. Número de publicaciones generadas

durante el periodo 2005-2011

El análisis por años muestra un comportamiento ascendente (gráfica 2) el número de artículos en revistas desde el año 2008 al 2011 ha tenido un crecimiento muy notorio, indicativo de que la temática está permeando en las líneas de investigación y que existe mayor interés en atenderla en las IES públicas oaxaqueñas. Se encontró una ausencia muy marcada de ensayos, capítulos de libro y libros sobre la temática lo que sugiere la urgencia de impulsar la producción académica de este tipo. Cabe aclarar que las tesis no figuran en la gráfica pues pertenecen al año 2013 y no es parte del periodo estudiado.

\section{Gráfica 2. Publicaciones generadas sobre emprendurismo}

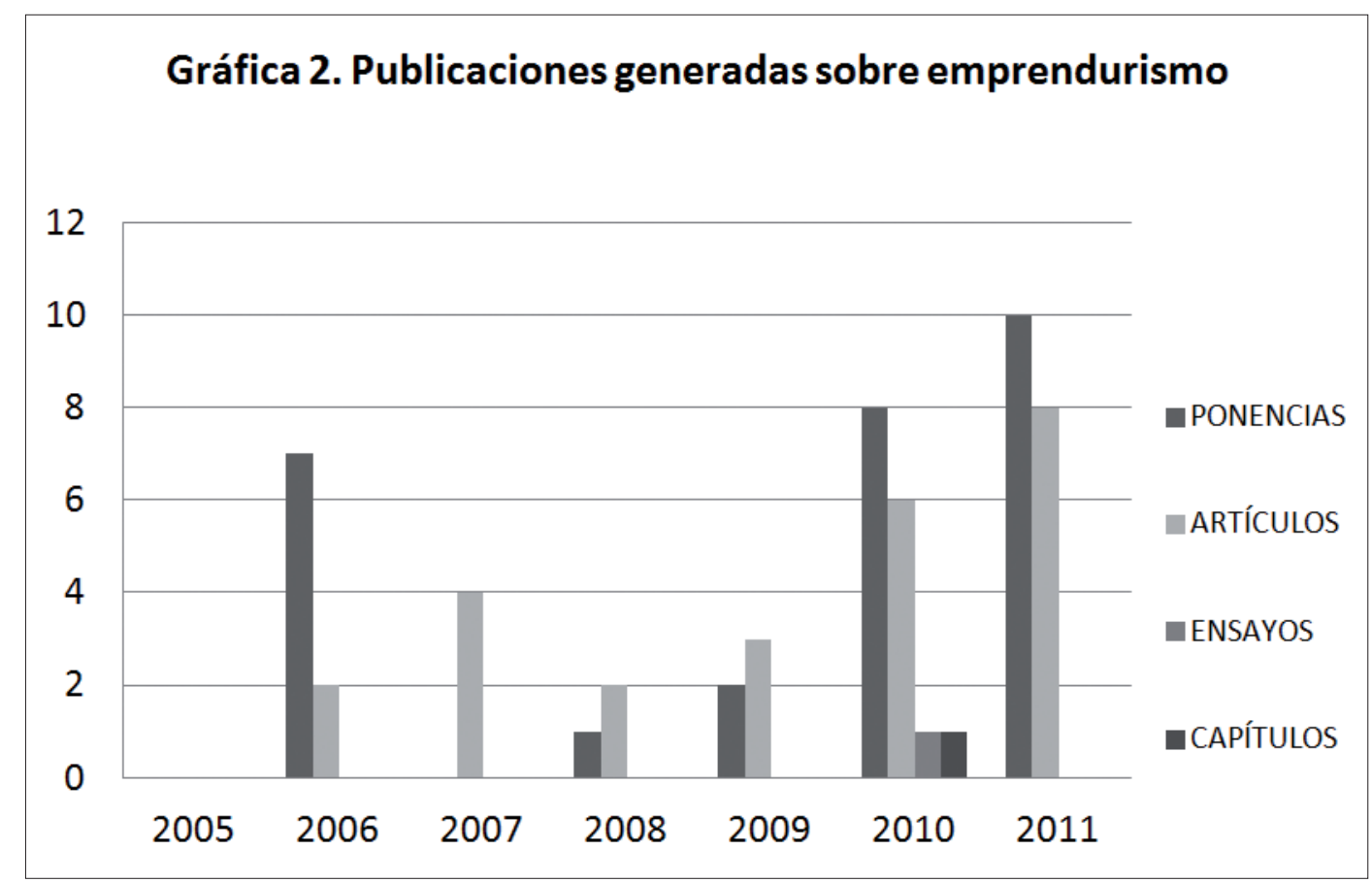

4. Tipos de eventos donde se han expuesto ponencias sobre emprendurismo

Al efectuar el análisis de los tipos de eventos académicos (gráfica 3), encontramos que los profesores investigadores han privilegiado la presentación de ponencias en Foros estatales en particular en el Foro Estatal de Investigación e Innovación promovido por el Consejo Oaxaqueño de Ciencia y Tecnología (Сосут), el 71\% de las ponencias generadas durante el periodo
2005-2011 se expusieron en estos eventos; en segundo lugar con un $15 \%$, se ubican los congresos, seminarios y coloquios nacionales y en menor proporción los congresos internacionales efectuados en la Universidad Autónoma de México y la Universidad Michoacana de San Nicolás de Hidalgo con un 15\% del total de las ponencias. Por lo anterior, sería recomendable la difusión del conocimiento generado en eventos de naturaleza internacional que permita a los investigadores oaxaqueños realimentarse 


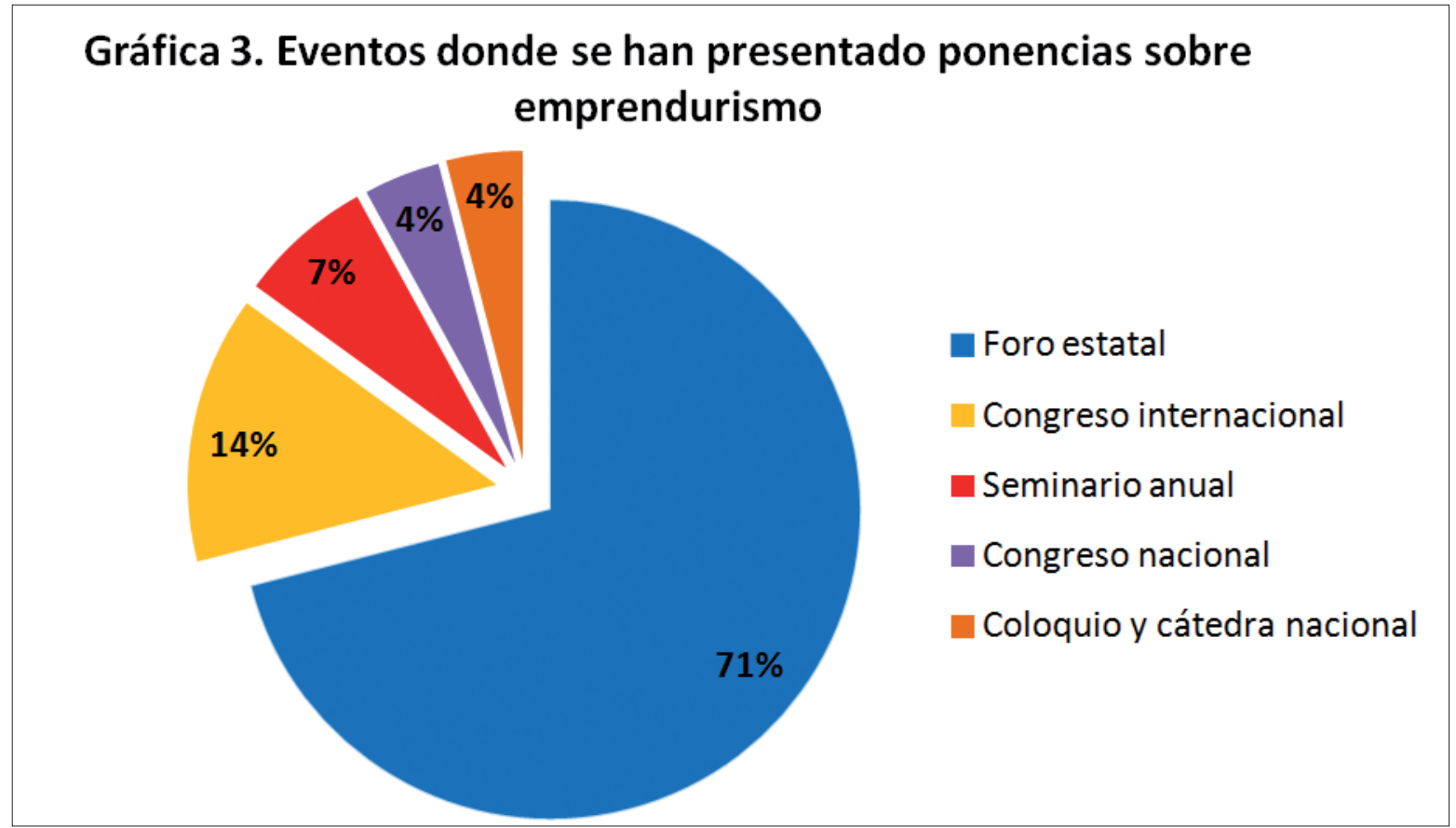

con los comentarios y experiencias de especialistas de otros estados del país o del extranjero y a la vez difundir sus trabajos en espacios con mayor radio de actuación.

\section{Campos temáticos y tipos de emprendurismo abordados en las investigaciones}

Al efectuar el análisis de los documentos según los trece campos temáticos que propone Villa y colaboradores (2009), se encontró que existen importantes vacíos por atender e investigar, la tabla 3 muestra que solo se han atendido nueve de los 13 campos y que los trabajos generados durante el periodo estudiado se han centrado en indagar sobre las buenas prácticas empresariales en distintos tipos y giros de negocios (23\%), los estudios de oportunidad para generar emprendimientos aprovechando los diversos recursos de las localidades (18\%), las condiciones económicas, cultu- rales y sociales en donde los diversos agentes llevan a cabo sus acciones de emprendimiento (13\%), al igual que los estudios sobre el tipo de organizaciones que crean los emprendedores oaxaqueños (13\%).

Escasamente se ha efectuado investigación referente a las temáticas de la educación para la formación de emprendedores (7\%) no obstante que en la mayoría de la IES ofertan programas educativos relacionados con el desarrollo de emprendedores, tampoco sobre los métodos para el impulso a la creación de empresas (7\%), sobre emprendimiento social (7\%), al igual que los estudios sobre emprendimiento y género $(7 \%)$ aunque no se puede negar el gran aporte hacia la economía familiar del emprendimiento femenino en sus diversas modalidades.

Existen cuatro campos temáticos que no han sido investigados: estudios o inves- 
tigaciones en el campo de la línea histórica, las fuentes de financiación para las nuevas empresas, los emprendimientos en empresas intensivas en tecnología y estudios comparativos, lo que representa una veta de oportunidades para trabajos futuros de los profesores investigadores oaxaqueños o de estudiantes de posgrado en un área del emprendurismo.

Agrupando a la producción académica de acuerdo a los cuatro tipos de emprendurismo (tabla 3 ), encontramos que más de tres cuartas partes de las investigaciones se han efectuado en el emprendurismo tradicional o de tipo lucrativo (81\%), lo que hace suponer que a los investigadores les interesa estudiar a las organizaciones (MiPyMES en todas sus modalidades) desde el punto de vista financiero o de rentabilidad económica; por otro lado y quizás atendiendo a la precaria situación socioeconómica y a la gran diversidad de recursos naturales con los que cuenta el estado de Oaxaca, es de interés que el 7\% de la producción académica sean estudios de emprendurismo social sobre todo en áreas de producción y comercialización de productos agropecuarios, de explotación de productos forestales no maderables y de lugares con mucho potencial para el desarrollo del ecoturismo. Sin embargo, escasamente se han efectuado estudios sobre emprendimiento femenino (7\%) y de tipo innovador (5\%) situación que proporciona a los investigadores grandes oportunidades para indagar en estas temáticas poco estudiadas en el Estado de Oaxaca.

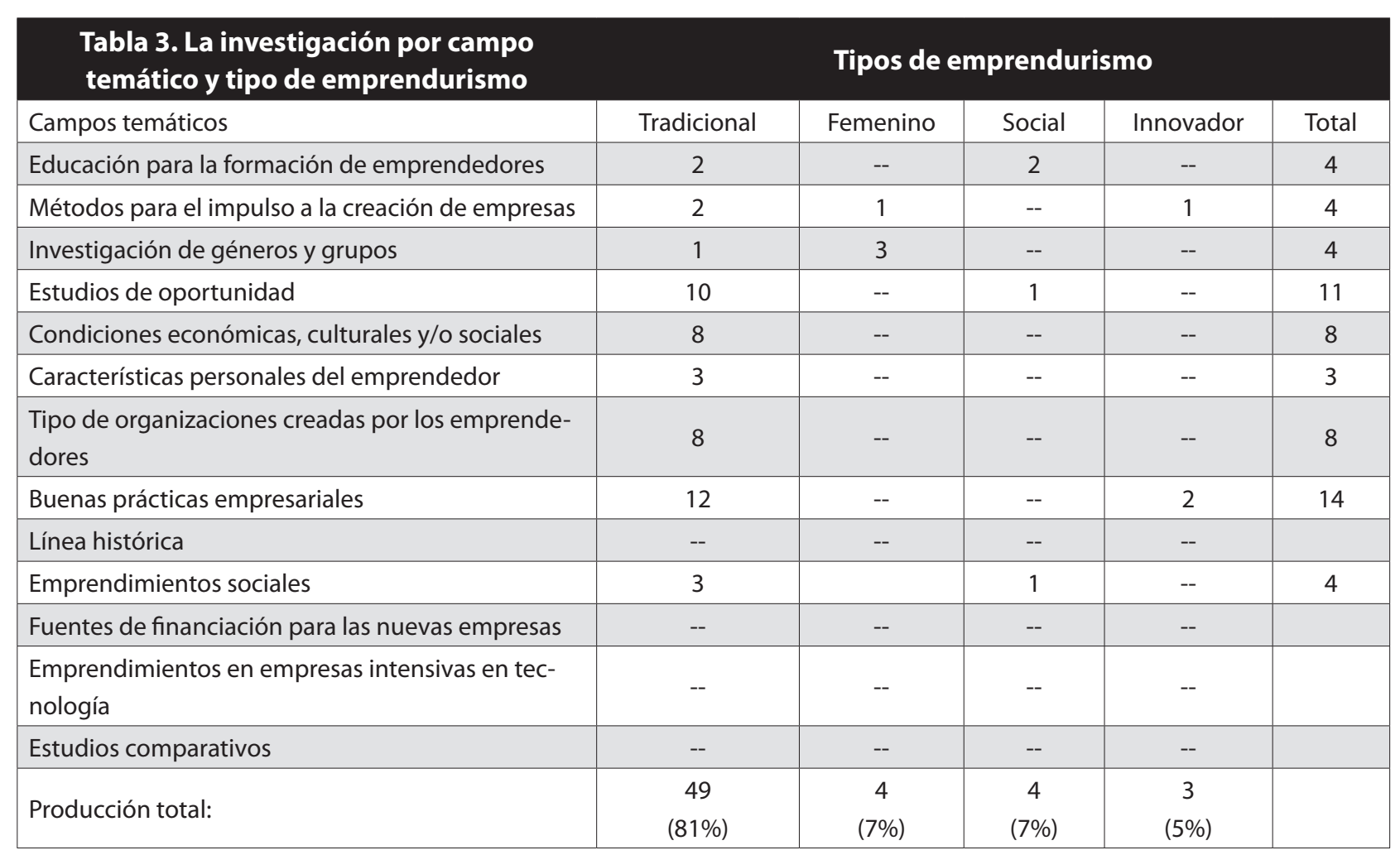




\section{Las problemáticas atendidas en las} investigaciones sobre emprendurismo

El análisis de toda la producción académica dio cuenta de catorce tipos de problemáticas de emprendurismo atendidas entre las que destacan cuatro: la primera (18\%) es la identificación de oportunidades de negocios a través de la comercialización de los recursos forestales no maderables, la producción y comercialización de árboles de navidad, el aprovechamiento del mármol y de recursos pétreos, la oportunidad del turismo religioso como impulsor y generador de fuentes de producción de diversos productos y el impulso de la hidroponía para generar alimentos e ingresos a las familias; la segunda problemática (17\%) es referida a la situación actual que enfrentan los negocios de artesanías para lo cual se analizan las estrategias de posicionamiento utilizadas, la integración de cadenas de valor, la creatividad e innovación, la orientación emprendedora y su relación con la eficacia de los negocios, así como otras tantas problemáticas del sector; el tercer grupo (13\%) lo constituyen las diversas situaciones que afrontan los sistemas de producción animal en diversas modalidades: aves de corral, cerdos, bovinos, piscicultura y meleagricultura, su forma de organización y su contribución en la generación de ingresos para los productores y sus familias; en cuarto lugar (8\%) atendiendo a la gran cantidad de atractivos turísticos del estado de Oaxaca las problemáticas se centran en el sector turístico abordando situaciones como la falta de información de lugares con potencial turístico no comunes, el desarrollo turístico mal planeado y sus diversas consecuencias en el medio ambiente de las comunidades rurales, las escasas capacidades de gestión empresarial y sustentabilidad que presentan los responsables de dirigir los centros ecoturísticos y las problemáticas de gestión empresarial que impiden el crecimiento y supervivencia de emprendimientos sociales ecoturísticos ubicados en poblaciones o lugares rurales.

\section{El marco teórico utilizado en la investigación en emprendurismo}

Se encontró una alta permeabilidad teórica en el tema del emprendimiento que son explicados por la formación académica de los investigadores autores de los trabajos (especialistas en agronomía, producción animal, administradores, economistas, educadores, entre otros) denotándose la especialización acumulada aludida por Harrison y Leitch (1996), la gran diversidad de fundamentos teóricos utilizados reflejan la aplicación de conocimientos disciplinares tan distintos a situaciones de emprendimiento mismos que serán de gran utilidad para empezar a construir un nuevo campo teórico del emprendurismo a partir de diversas aproximaciones multidisciplinarias (Bygrave, 1989). Los únicos trabajos que aluden directamente a la teoría tradicional del emprendurismo tales como el concepto mismo, su evolución y su importancia, entre otros, fueron aquellos que estudiaron el fenómeno de la educación y el emprendurismo y que constituyen apenas el 7\% del total de la producción académica analizada. 
REVISTA DE INVESTIGACIÓN EDUCATIVA DE LA REDIECH N. 9 ISSN: 2007-4336

\section{La metodología empleada en la investigación en emprendurismo}

La mayoría de los trabajos (43\%) fueron de tipo descriptivo exploratorio con un enfoque netamente cuantitativo lo que bien puede indicar que la investigación en emprendurismo en las IES públicas oaxaqueñas está en proceso de formación y que los resultados obtenidos de éstas pueden abrir interesantes vetas de investigación para profundizar en la temática en el futuro; de igual forma el enfoque metodológico que se privilegia en la mayoría de los casos se desarrolla a partir de la aplicación de cuestionarios diseñados ex profeso y en otros mediante instrumentos estandarizados como el Serviqual.

Cabe aclarar que en la mayoría de los trabajos la información de los cuestionarios fue complementada con información obtenida mediante entrevistas no estructuradas y en algunos casos de tipo informal o mediante la observación (aunque no se menciona el diseño de guías de observación). Llama la atención el hecho de que el $28 \%$ de los trabajos fueron descriptivos exploratorios en los que se utilizó una metodología cuanti-cualitativa o mixta lo que representa una riqueza en los resultados al efectuarse una triangulación lo que bien pudiera denotar un avance en cuanto a la estructuración de un marco metodológico más enriquecedor. En algunas investigaciones se aplicaron metodologías específicas con mucha tradición tales como las técnicas de sondeo rural participativo, la matriz de contabilidad social, la metodología FODA, diagnóstico administrativo, entre las más destacadas.
De especial interés son las aportaciones de cuatro trabajos que son pioneros en los estudios de emprendurismo al utilizar una metodología de tipo experimental para demostrar cómo se puede incrementar la rentabilidad económica de los productos agrícolas cuando se implementan mejoras en el sistema de producción, en el peso de aves de corral y en la producción de huevos, así como la aplicación de la técnica "perfil flash" para la evaluación sensorial de carnes.

9. Las tesis, hipótesis, supuestos y/o preguntas de investigación en la producción en emprendurismo

En el análisis de los 60 trabajos se identificaron de manera explícita e implícita las hipótesis, preguntas de investigación, tesis y supuestos. Se encontró que las hipótesis y las preguntas de investigación planteadas están bien relacionadas con los objetivos propuestos en las investigaciones. Quince trabajos (25\%) presentan hipótesis aunque llama la atención que en su mayoría no son propiamente de naturaleza cuantitativa y en sus resultados y conclusiones no muestran si dichas hipótesis se aceptan o rechazan quedándose a nivel de trabajos de corte descriptivo; nueve trabajos (15\%) presentan preguntas de investigación mismas que se responden apropiadamente en los resultados o conclusiones; cabe aclarar que trece trabajos (22\%) presentaron de manera implícita tesis o supuestos ya que no fueron redactados de manera puntual por los investigadores y fue durante el análisis de los documentos que fue posible identificarlos. Sin embargo, se encontraron debilidades en la investigaciones, como el 
hecho de que 23 documentos (38\%) de la producción no presentó hipótesis, preguntas de investigación, tesis ni supuestos ya que estos elementos constituyen un mapa o guía para el trabajo de investigación, razón por la cual sería recomendable conocer la formación o habilitación en investigación científica de los autores de dichos trabajos.

10. Principales resultados obtenidos en la producción en emprendurismo

Si bien es cierto que los hallazgos reportados en la producción académica analizada son variados sólo nos detendremos en aquellos tres grupos de resultados que son más representativos. Puesto que se ha estudiado mucho la problemática del sector de artesanías se han obtenido abundantes resultados sobre la situación que guardan los negocios y/o talleres de artesanías en aspectos relacionados con la innovación en la cadena de valor, las ventajas competitivas, los factores de éxito, las fuentes creativas de conocimiento que permiten posicionar al sector de artesanías en el mercado entre otros.

En un segundo grupo se ubican los resultados obtenidos sobre el gran potencial que ofrecen algunos recursos para generar oportunidades de negocio en comunidades rurales como es el caso del aprovechamiento de los recursos forestales no maderables, los agregados pétreos, el turismo religioso como centro impulsor de la creación de negocios relacionados con dicha actividad (servicios de alimentación y hospedería, artesanías, entre otros) y las actividades relacionadas con el turismo al ubicar oportunidades de negocios mediante la elabora- ción de un inventario de lugares con gran potencial ecoturístico en el estado, así como mejorar la administración y gestión de los centros ya establecidos en beneficio de los pobladores de las comunidades y en tercer lugar, se obtuvieron resultados interesantes sobre la situación que guardan diversos sistemas de producción, así se identificó que los sistemas de producción avícola, porcina, bovina y piscícola son generalmente explotaciones de traspatio caracterizadas por ser altamente tradicionales y para autoconsumo, no obstante dichas actividades tienen potencial para generar ingresos adicionales a los productores si estos pudieran identificar canales de comercialización adecuados.

\section{Conclusiones}

Los resultados obtenidos de este acercamiento exploratorio sobre el estado del arte de la investigación en emprendurismo nos permiten llegar a las siguientes conclusiones:

1) Es necesario impulsar y fortalecer la investigación en emprendurismo en el estado de Oaxaca pues el 63\% de la producción académica analizada se concentra en solo cuatro (12\%) de las 34 IES públicas del estado en el seno de los programas de posgrado que ofertan, mientras que los Institutos Tecnológicos tienen una escasa o nula participación a pesar de ofertar licenciaturas en administración de empresas, desarrollo e innovación de negocios y otras áreas afines, lo que representa una debilidad ante las nuevas exigencias de las políticas educativas nacionales e internacio- 
nales de impulsar el desarrollo del espíritu emprendedor en las nuevas generaciones de universitarios.

2) Se observa una desigualdad en la distribución de los investigadores según el género pues más de dos terceras partes son hombres y el resto mujeres, lo que bien podría explicar la escasa investigación en temas de emprendimiento femenino.

3) Las temáticas más abordadas en la investigación se concentran en cuatro grupos: las buenas prácticas empresariales, los estudios de oportunidad de negocios, las condiciones económicas, culturales y/o sociales donde surgen los emprendimientos y el tipo de organizaciones creadas por los emprendedores; por lo anterior, se presentan oportunidades para investigar emprendurismo en cuatro temáticas que no han sido atendidas: la línea histórica de empresas, las fuentes de financiamiento para nuevas empresas, los emprendimientos en empresas intensivas en tecnología y los estudios comparativos.

4) Las investigaciones en su mayoría estudian al emprendurismo tradicional o lucrativo (81\%), al emprendurismo social (7\%), descuidando al emprendurismo femenino y al emprendurismo innovador, ambos de gran importancia para aprovechar las riquezas naturales y contribuir al desarrollo regional del estado de Oaxaca.

5) La producción académica sobre emprendurismo debe ser difundida en revistas de prestigio indexadas e impulsar la publicación en forma de capítulos de libros o libros pues no existen a la fecha; de igual forma las ponencias sobre la temática deben ser presentadas - para una mayor difusión- en eventos de carácter nacional e internacional y no quedarse sólo en el ámbito local o estatal.

El estado del arte efectuado muestra que la temática de emprendurismo en el estado de Oaxaca se encuentra en una etapa embrionaria de desarrollo y que es urgente que las IES públicas tomen un papel protagónico y atiendan a esta temática emergente de investigación educativa. Ante los resultados obtenidos cabe preguntarnos cuáles son las actividades futuras que pueden surgir como una agenda pendiente para impulsar esta temática de investigación en el estado de Oaxaca; pueden existir varias respuestas que nos lleven a la realización de diversas acciones y actividades entre las cuales destacan las siguientes:

1) Al haber ubicado a los investigadores y a sus IES de adscripción será posible establecer contactos e integrar redes de colaboración (Red Oaxaca de Investigadores en Emprendurismo) llevar a cabo seminarios e investigación de frontera y de alto impacto en la temática (En este sentido como parte de las actividades del proyecto de investigación y para socializar los resultados del mismo, el Cuerpo Académico "Estudios Multidisciplinarios" organizó y llevó a cabo en el mes de julio del año 2014 el Primer Encuentro Regional de Investigación Multidisciplinaria con tres mesas de trabajo: Emprendurismo, Educación y Género).

2) Socializar los resultados de la investigación con organismos públicos estatales como el Consejo Oaxaqueño de Ciencia 
y Tecnología y con los responsables de las IES públicas oaxaqueñas que estén planeando ofertar programas de posgrado en áreas de Gestión de Negocios y Desarrollo Regional, para que tomen en cuenta los cinco campos temáticos en emprendurismo que a la fecha no han sido investigados a fin de que establezcan, cultiven y desarrollen líneas de investigación y contribuyan mediante las tesis de maestría y doctorado con la generación de conocimiento y la formación de recursos humanos en dichos campos.

3) Considerando la gran diversidad de recursos naturales del estado de Oaxaca los resultados muestran interesantes vetas para investigar sobre emprendurismo innovador y emprendurismo social a fin de proponer formas de aprovechar dichos recursos que se traduzcan en mejoras en la calidad de vida de los habitantes de las comunidades.

4) Atendiendo al impulso actual de las políticas públicas para incrementar la participación de la mujer en la contribución de la economía, existen grandes oportunidades para desarrollar investigaciones en emprendurismo femenino pues no existe información en nuestro estado sobre las situaciones que enfrentan las mujeres que deciden emprender sus negocios.

Por último, como integrantes del Cuerpo Académico "Estudios Multidisciplinarios", estamos conscientes de las limitantes que puede presentar cualquier estado del arte -la nuestra no escapa a este hecho- al no considerar toda la producción académica existente debido a las múltiples dificultades para poder acceder a ella, sin embargo, creemos que es un primer acercamiento a la temática y que posiblemente seamos un referente para los colegas que quieran continuar en esta línea de investigación.

\section{Bibliografía}

Bygrave, W. (1989). The entrepreneurship paradigm: a philosophical look at its research methodologies en Entrepreneurship Theory and Practice, 14 (1), 7-26.

Casson, M. (1982). The Entrepreneur. An Economic Theory. Oxford: Martin Robertson.

CE (2004). Ayudar a crear una cultura empresarial. Guía de buenas prácticas para promover las actitudes $y$ capacidades empresariales mediante la educación. Luxemburgo: Comisión Europea/Dirección General de Empresa.

Coстт (2006). Memorias del $10^{\circ}$ Foro Estatal de Investigación Científica y Tecnológica Oaxaca 2006. Consejo Oaxaqueño de Ciencia y Tecnología.

(2010). Memorias del 12 Foro Estatal de Investigación e Innovación Oaxaca 2010. Consejo Oaxaqueño de Ciencia y Tecnología.

(2011). Memorias del $13^{\circ}$ Foro Estatal de Investigación e Innovación Oaxaca 2011. Consejo Oaxaqueño de Ciencia y Tecnología.

Damián Simón, J. (2013). Sistematizando experiencias sobre educación en emprendimiento en escuelas de nivel primaria en Revista Mexicana de Investigación Educativa. Consejo Mexicano de Investigación Educativa. 18(56), pp. 159-190.

De Pablo, I. (2005). El emprendizaje social: motor de desarrollo y cohesión social. V Seminario sobre creación de empresas y entorno. Universidad Autónoma de Madrid. Trujillo.

Fuentes, F. y Sánchez, S. (2010). Análisis del perfil emprendedor: una perspectiva de género. Revista Estudios de Economía Aplicada. Vol. 8-3 pp.1-28.

García, José M.; Villanueva, M. y Collado, J. (2005). TouriSME: Emprendurismo en las Pequeñas y Medianas Empresas. Comunidad Valenciana, North East of England, Sachsen-Anhalt y, Limburg. Universidad Politécnica de Valencia. Valencia.

González, F.; Peña, M. y Vega, Z. (2010). Formación emprendedora en el contexto de la Universidad Centro Occidental Lisandro Alvarado. Revista científica di- 
gital del Centro de Investigación y Estudios Gerenciales. 1(2). Pp. 11-31.

Gutiérrez G. (2011). El comportamiento emprendedor en el Salvador. Universidad de Cádiz. Departamento de Organización de Empresas.

Harrison, R. y Leitch, C. (1996). Discipline emergence in entrepreneurship: accumulative fragmentalism or paradigmatic science? Entrepreneurship, Innovation and Change, 5 (2). pp 65-83.

Kihilstrom, R. y Laffont, J. (1979). A general equilibrium entrepreneurial - theory of firm formation based on risk aversion. Journal of Political Economy, 87, pp.719-748.

Kelley, D., Bosma, N. y Amorós J. (2011). Global Entrepreneurship Monitor. 2010 Global Report. BabsonUniversidad del Desarrollo. Santiago de Chile.

Martínez Rodríguez, M. (2008). Análisis de competencias emprendedoras del alumnado de las Escuelas Taller y Casas de Oficios en Andalucía. Primera fase del diseño de programas educativos para el desarrollo de la cultura emprendedora entre los jóvenes. Universidad de Granada. Editorial de la Universidad de Granada.

Pérez, T. (2010). Iniciativa emprendedora en la educación en España, estado de situación y propuestas. Ministerio de Industria, Turismo y Comercio.

Reyes, M.; Zilleruelo, C. e Hidalgo, G. (2012). Lo que Centroamérica puede hacer para que el emprendimiento dinámico apoye su (nuevo) crecimiento. Mapeo y análisis de Ecosistemas en siete países. Santiago de Chile. Banco de Desarrollo de América Latina.

Valls, N., Villa, A., Martínez, S. y Hernando, A. (2009). Emprendimiento Social Juvenil. 18 buenas prácticas. Barcelona. Fundación Bertelsmann.

Veciana, M. (1999). Creación de empresas como programa de investigación científica. Revista Europea de Dirección y Economía de la Empresa. 8(3), pp. 1136.

Villa, M.; Alvarez, K.; Toro, M. y Piemontese, A. (2007). Estado del arte de la investigación en emprendimiento empresarial en la universidad iberoamericana durante los años 2000 a 2004. Revista Politécnica, vol. 3, (5), p. 35-46.

Watson, H. (2001). Small Business Versus Entrepreneurship Revisited. Brockhaus, Robert H., Gerald E. Hills, Heinz Klandt, \& Harold P. Welsch, (editors), Entrepreneurship Education: A Global View. Aldershot: Ashgate. 